\title{
然
}

\author{
Carlos J. Tórtola Sebastián*
}

Neus Alfaraz Hernández**

\section{EL IMPACTO DE LA COVID-19 EN LA ECONOMÍA CHINA}

La epidemia de la COVID-19 se originó en China a finales de diciembre de 2019, convirtiéndose en una crisis global sin precedentes tres meses después. La crisis sanitaria generó un doble shock negativo de oferta y demanda en la economía china que causó su primera tasa de crecimiento negativa desde 1976. Por su parte, la expansión mundial de la epidemia supuso la congelación de su demanda externa. En este contexto, las autoridades han aplicado numerosas medidas de estímulo desde el inicio de la epidemia, estableciendo como prioridad la estabilidad del mercado laboral en detrimento de un objetivo de crecimiento económico en 2020. Pese a los desafíos pendientes, China será el primer país en superar la crisis y uno de los pocos que se prevé que logre una tasa de crecimiento positiva este año. Por ello, su ejemplo puede servir como referencia al resto de economías en su lucha contra la COVID-19.

Palabras clave: crisis económica, coronavirus, pandemia, shock oferta, shock demanda, política económica.

Clasificación JEL: H12, O53.

\section{Introducción}

La pandemia de la COVID-19 se ha convertido en el mayor shock negativo de la economía mundial desde la Gran Depresión de 1929, superando ampliamente el impacto negativo generado por otras graves crisis ocurridas durante las últimas décadas, como la crisis de las empresas puntocom de comienzos del siglo XXI

\footnotetext{
*Técnico Comercial y Economista del Estado.

**Diplomada Comercial del Estado.

Las opiniones expresadas solo comprometen a sus autores.

Versión de junio de 2020

DOI: https:/doi.org/10.32796/bice.2020.3125.7041
}

- la crisis financiera de 2008. El brote de COVID-19 tuvo su origen en China a finales de 2019 y el 10 de marzo de 2020 ya se había extendido a 115 países.

China fue el primer país en sufrir el brote de COVID-19 y está siendo también el primer país en superar esta crisis sanitaria. Por ese motivo, la evolución de la epidemia en este país constituye una referencia para el resto del mundo que debe ser cuidadosamente analizada. Asimismo, China es una de las principales economías del mundo, con una participación en el PIB mundial del $19,2 \%$ y del $12,4 \%$ en el comercio mundial, por lo que el impacto y $\triangleright$ 
desarrollo de cualquier crisis que sufra el país afecta directamente a la evolución de la economía global, lo que justifica aún más este análisis.

Este artículo analiza, en primer lugar, la evolución de la epidemia de COVID-19 en China. A continuación, se centra en su impacto económico, diferenciando entre el impacto inicial y la evolución experimentada en los meses posteriores por la economía china. En un tercer apartado, se desgranan las distintas políticas económicas aplicadas por las autoridades chinas. Por último, se concluye indicando cómo podría evolucionar la economía china en 2020.

\section{Evolución de la COVID-19 en China}

Los primeros casos de COVID-19 se detectaron en la ciudad china de Wuhan (provincia de Hubei) a finales de diciembre de 2019. Sin embargo, no fue hasta el 20 de enero cuando China reconoció ante la Organización Mundial de la Salud (OMS) su gravedad. A diferencia de lo ocurrido con las epidemias del SARS y MERS, el 11 de marzo la OMS elevaba la enfermedad a nivel de pandemia debido a su vertiginosa propagación a nivel mundial (OMS, 2020a).

El brote de COVID-19 en China coincidió con la festividad del Año Nuevo chino, que este año abarcaba desde mediados de enero hasta la primera semana de febrero. Su rápida expansión por todo el país obligó al Gobierno a retrasar la incorporación al trabajo, hasta el 10 de febrero en el caso de Pekín, ordenando una vuelta escalonada a las ciudades y centros de producción para controlar el riesgo de contagio (Municipalidad de Pekín, 2020a).

Paralelamente, se aplicaron drásticas medidas de control y prevención a nivel nacional que incluían el confinamiento de ciudades (obligatorio en la provincia de Hubei, cuya población roza los 60 millones) (Gobierno Provincial de Hubei, 2020); el cierre de comercios, negocios, centros educativos, oficinas, fábricas y centros turísticos; el desarrollo de servicios de entrega a domicilio y el establecimiento del teletrabajo; controles de temperatura en lugares abiertos al público (i.e., aeropuertos, estaciones de ferrocarril y autobús, supermercados, centros comerciales, viviendas); y la obligatoriedad de cuarentenas de catorce días a cualquier persona de nacionalidad china o extranjera que regresase a su lugar de trabajo tras el periodo vacacional (Municipalidad de Pekín, 2020b).

Por su parte, las autoridades locales comenzaron a desarrollar planes específicos de inspección de los centros de trabajo y producción, en función del nivel de riesgo de sus respectivas provincias, y a exigir la instalación de sistemas de control y prevención adecuados para reiniciar la actividad económica a la mayor brevedad (Consejo de Estado de China, 2020a).

Estas medidas comenzaron a dar sus frutos a mediados de febrero, cuando se inició una tendencia a la baja en el número diario de infectados. La visita sorpresa del presidente chino Xi Jinping a la ciudad de Wuhan, el 10 de marzo, contribuyó a reforzar el mensaje oficial de que China estaba dejando atrás la epidemia (Baijie, 2020). Paradójicamente, los primeros signos de estabilización del coronavirus en el país coincidieron con su propagación en el exterior durante el mes de marzo, desplazándose el epicentro de la epidemia de China a Europa (Ren, 2020), lo que sitúa la evolución de la COVID-19 en China 45 días por delante de los países europeos (Gráfico 1).

A partir de marzo, comercios y empresas retomaron gradualmente su actividad bajo $D$ 
ciertos controles por temor a un repunte del brote, como ocurrió en algunas provincias tras la vuelta de trabajadores chinos y viajeros procedentes del exterior. Pese a este clima de incertidumbre, que impidió a las autoridades llevar a cabo una relajación total de las políticas de contención, el $95 \%$ de las grandes empresas y el $60 \%$ de las pymes fuera de la provincia de Hubei habían reiniciado su actividad a principios de marzo mediante la alternancia del teletrabajo con turnos presenciales en las empresas y las Administraciones públicas para evitar aglomeraciones (Y. Cheng, 2020).

En abril, el continuo descenso de nuevos casos permitió al Gobierno modificar su estrategia de gestión de la epidemia, fijando la prioridad en el control de los casos importados y extendiendo la relajación parcial de las medidas de control para favorecer la reactivación económica del país. A modo de ejemplo, la apertura de algunos restaurantes y tiendas quedaba sujeta al cumplimiento de estrictas medidas de higiene y control del aforo.

En este escenario de estabilización, el macropuente del Día del Trabajo (del 1 al 5 de mayo) supuso la primera prueba de fuego para las autoridades en su intento de reactivar la economía. A partir del 30 de abril los Gobiernos locales comenzaron a levantar las restricciones a la movilidad para viajar dentro del país, a excepción de algunas provincias del noreste donde aún existía un riesgo elevado de contagio. En esos cinco días se realizaron 115 millones de desplazamientos con un gasto superior a los 6.700 millones de dólares. Pese a constituir una disminución de casi 10.000 millones de dólares respecto a 2019, estas cifras se interpretaron como un paso adelante en el proceso de normalización de la actividad económica del país (Wang, 2020). A lo largo del mes de mayo se fueron aprobando otras medidas relevantes para garantizar la vuelta a la normalidad, como la autorización para retomar la actividad en todas las tiendas, restaurantes y hoteles que apliquen medidas de precaución; la reapertura de instalaciones recreativas como cines, gimnasios, museos y parques a un número limitado de asistentes; y la eliminación del uso de mascarillas en espacios abiertos si se cumple una distancia social de $D$

CASOS DIARIOS REGISTRADOS POR COVID-19

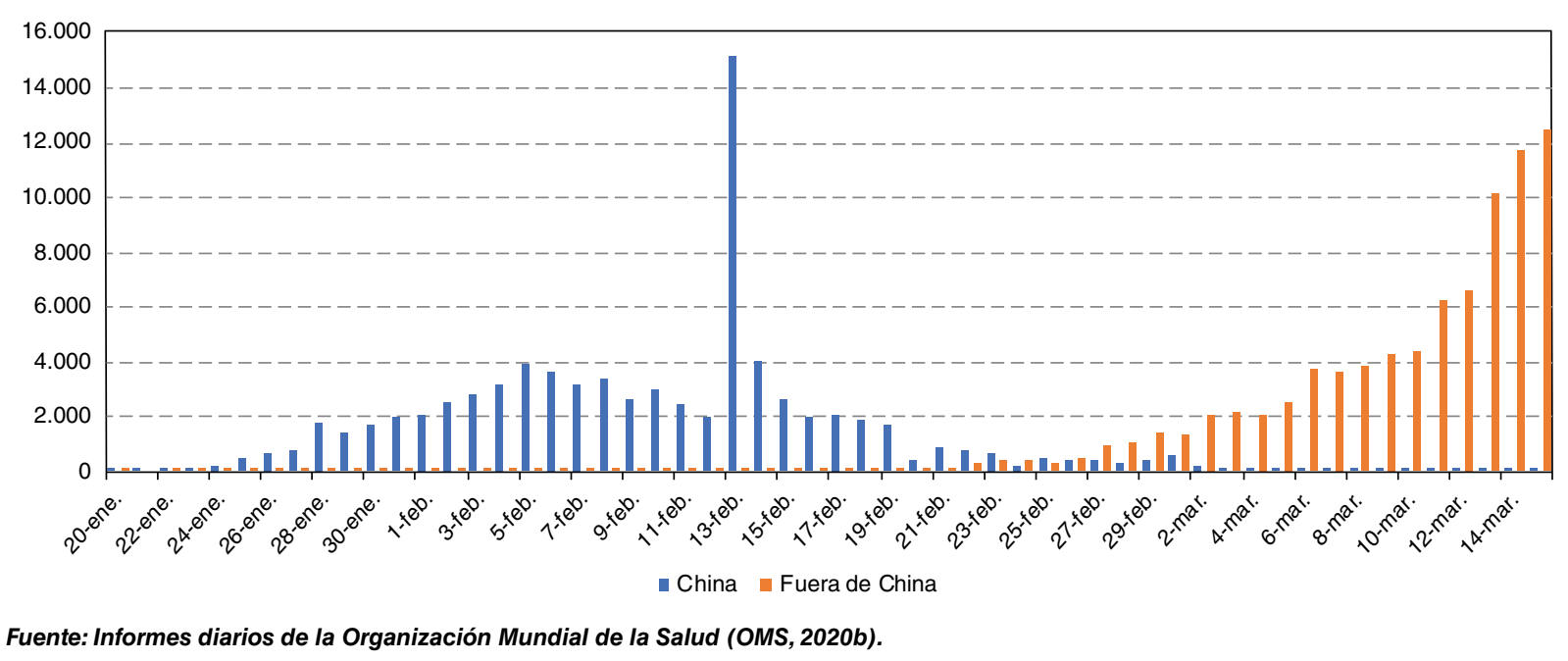


seguridad (Consejo de Estado de China, 2020b; S. Cheng, 2020).

Se puede afirmar, por tanto, que en el mes de junio China ha recuperado la actividad habitual, con la particularidad de la integración en la vida cotidiana de ciertas medidas de prevención que se mantendrán hasta que las autoridades puedan garantizar el control total sobre la COVID-19, lo que dependerá no solo de sus políticas internas, sino también de la evolución y gestión de la epidemia a nivel mundial.

\section{Impacto de la COVID-19 en la economía china}

Las drásticas medidas de contención de la COVID-19 adoptadas por las autoridades del país han provocado un doble shock negativo en la economía china durante los primeros meses de 2020. Por el lado de la demanda, las restricciones a la movilidad de la población, el aumento de la incertidumbre y el comportamiento precautorio de los agentes económicos redujeron el gasto de los hogares. Mientras que, por el lado de la oferta, las estrictas limitaciones a la movilidad de la población y el cierre (total o parcial) de fábricas y oficinas provocaron la ruptura de la cadena global de suministro. Fruto de este doble shock, el producto interior bruto (PIB) del país se contrajo un $6,8 \%$ en el primer trimestre de 2020 , lo que supuso la primera tasa de crecimiento negativa de la economía china desde 1976 (Gráfico 2) (NBSC, 2020).

Pese a ello, a medida que se empezaron a relajar las medidas de contención, se inició una mejora progresiva de los indicadores económicos, de manera que el Fondo Monetario Internacional (FMI), en sus previsiones macroeconómicas de abril, estimó que China será una de las pocas economías que evitará la recesión en 2020 con un crecimiento del $1,2 \%$ a finales de año, lejos del $6,1 \%$ alcanzado en 2019 (IMF, 2020; NBSC, 2020). Como resultado de esta incertidumbre y las bajas $\triangle$

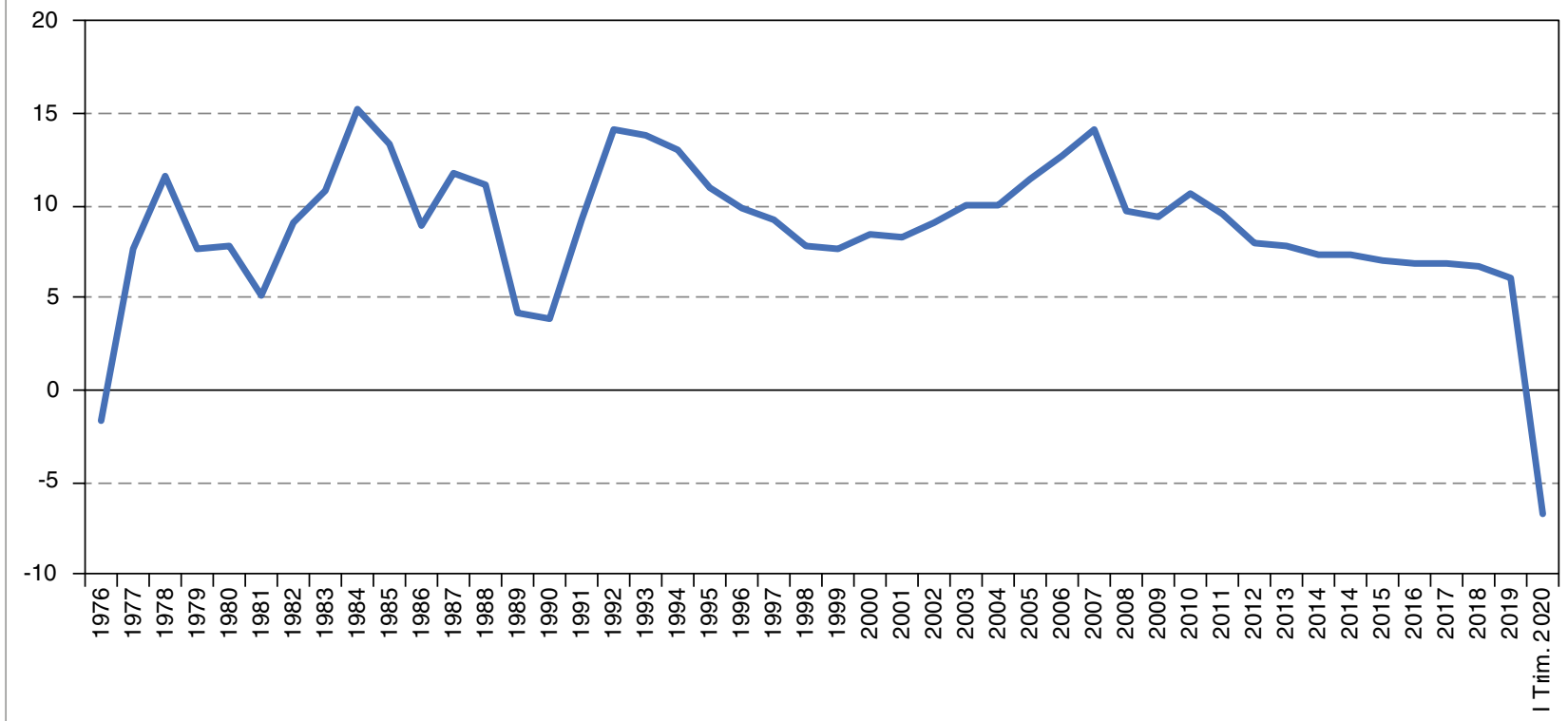


expectativas de crecimiento que existen para 2020 , por primera vez en treinta años las autoridades chinas no han fijado ningún objetivo oficial de crecimiento anual durante la celebración de las «Dos Sesiones», el gran evento político del país donde se anuncian los objetivos de política económica y social para el año correspondiente.

\subsection{Impacto de la COVID-19 por el lado de la demanda}

Como se ha mencionado, el inicio de la epidemia de la COVID-19 en China coincidió con la celebración del Año Nuevo chino, tradicionalmente uno de los periodos de mayor gasto en el país (Lee, 2020). En el primer trimestre de 2020 , el gasto en consumo de los hogares se contrajo un $12,5 \%$ interanual, frente al crecimiento del $5,4 \%$ en el mismo periodo de 2019 , consecuencia del confinamiento de la población, las restricciones a la movilidad dentro del país y el cierre obligatorio de comercios, canal HORECA y centros de ocio (NBSC, 2020).

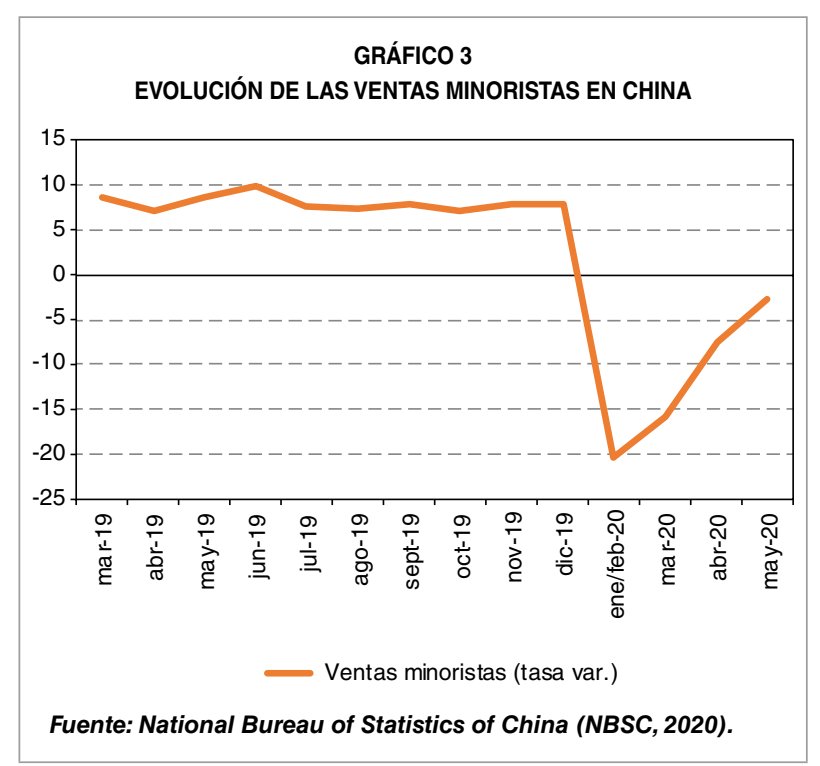

Dentro del descenso del consumo de los hogares destacó la caída de las ventas minoristas de bienes en un 20,5\% interanual en el periodo enero-febrero ${ }^{1}$, experimentando una progresiva mejoría con un descenso del 15,8\% en marzo, el $7,5 \%$ en abril y el $2,8 \%$ en mayo debido a la suavización de las medidas de contención (Gráfico 3) (NBSC, 2020). Por categorías, las ventas de automóviles, uno de los principales motores de la economía china, se derrumbaron un $79 \%$ en febrero, seguido de un $-43 \%$ en marzo y un crecimiento del $4,4 \%$ en abril (CAAM, 2020a y 2020b). Sin embargo, la mayor contracción del gasto de los hogares se produjo en el sector servicios al requerirse la presencia física del consumidor, siendo las categorías más afectadas durante el primer trimestre de 2020 educación, cultura y ocio (-36,1\% interanual), transporte y comunicación (-17\% interanual), y atención médica y servicios de salud $(-10,2 \%$ interanual) (NBSC, $\triangleright$ 2020).

1 Las autoridades chinas publicaron los datos económicos de enero y febrero de manera conjunta para eliminar la volatibilidad del Año Nuevo chino.

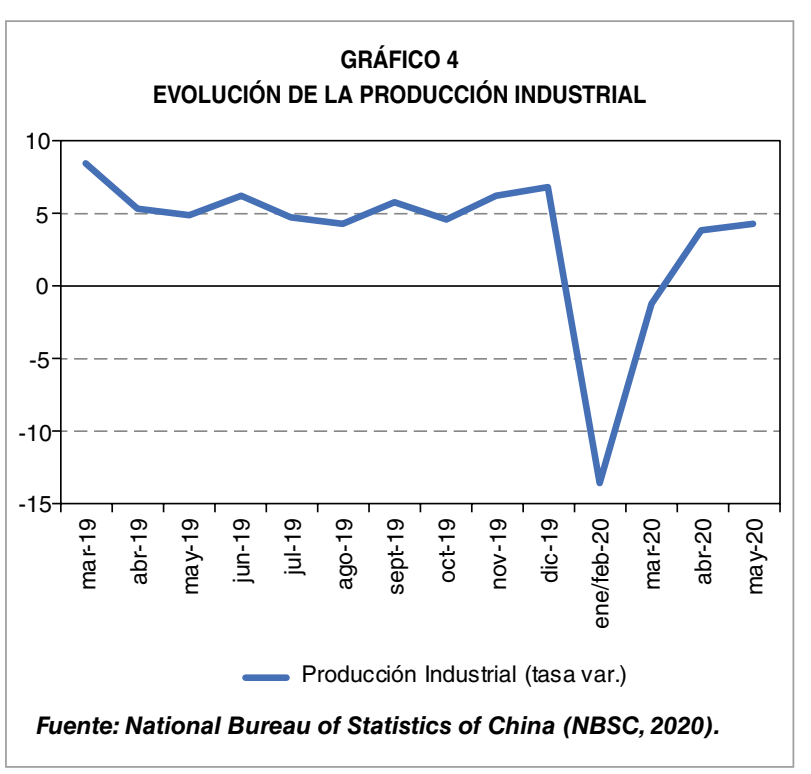


La apertura progresiva de comercios y establecimientos a partir de marzo fue insuficiente para la recuperación del consumo interno debido a la persistencia del comportamiento precautorio entre la población por temor a un segundo brote (Mazor-Freedman, 2020). En este clima de desconfianza el comercio online de bienes (p.e., alimentos frescos, bienes de consumo) y servicios (p.e., educación a distancia, consultas médicas online) recibió un fuerte respaldo por parte de los consumidores frente a las ventas presenciales, con un aumento del $0,8 \%$ interanual en el primer trimestre, que se confirmó con un crecimiento del 4,5\% interanual en los cinco primeros meses del año (NBSC, 2020). El protagonismo del comercio online durante el brote de la COVID-19 queda reflejado en el incremento en un $22 \%$ interanual de la cifra de negocio de Alibaba durante el primer trimestre del año (La Monica, 2020). De consolidarse esta tendencia en el resto de las plataformas de e-commerce en los próximos meses, la aceleración del proceso de digitalización del comercio se convertiría en uno de los principales cambios estructurales en la economía china causados por la COVID-19.

Respecto a la inversión en activos fijos por parte de los hogares ${ }^{2}$, esta cayó un $24,5 \%$ en los dos primeros meses de 2020, un 16,1\% interanual en el primer trimestre, recuperándose hasta el $-10,3 \%$ en los cuatro primeros meses y al $-6,3 \%$ entre enero y mayo. Las ventas inmobiliarias, componente principal de la deuda de los hogares con un crecimiento anual medio del $22 \%$ entre 2015 y 2019 , experimentaron una reducción del 16,3\% interanual entre enero y febrero, iniciando una progresiva mejora a medida que se relajaron los protocolos de contención a partir de marzo con un descenso del

2 La inversión en activos fijos excluye a los hogares rurales.
$7,7 \%$ en el primer trimestre, del $3,3 \%$ en el periodo entre enero y abril, y del $0,3 \%$ entre enero y mayo (García Herrero et al., 2020; NBSC, 2020).

\subsection{Impacto de la COVID-19 por el lado de la oferta}

Por el lado de la oferta, el cierre obligatorio de los centros de producción a mediados de enero generó una caída del valor agregado industrial del $-13,5 \%$ en los dos primeros meses ${ }^{3}$ de 2020 , seguido de una progresiva recuperación del $-1,1 \%$ en marzo, el 3,9\% en abril y el $4,4 \%$ en mayo, a medida que se producía la reapertura controlada de las fábricas (Gráfico 4) (NBSC, 2020). El Índice de Gestores de Compras (PMI) del sector manufacturero, indicador del sentimiento de mercado entre las empresas del sector ${ }^{4}$, se desplomó en febrero hasta los 35,7 puntos, mejorando en marzo hasta los 52 puntos para descender de nuevo a los 50,8 puntos en abril. El motivo del descenso del PMI en abril se debió a la caída del componente de pedidos de exportación, que se redujo hasta los 33,5 puntos, frente a los 46 puntos de marzo, debido a la congelación de la demanda externa por la expansión internacional de la epidemia. En mayo, el PMI se situó en 50,6 puntos (Gráfico 5) (NBSC, 2020).

Esta mejora de la actividad industrial en China, resultado de la reapertura de sus fábricas y parques industriales, se produjo de manera progresiva y con las empresas operando lejos de su nivel óptimo de producción. Esto $\triangleright$

\footnotetext{
3 Las autoridades chinas publicaron los datos económicos de enero y febrero de manera conjunta para eliminar la volatibilidad del Año Nuevo chino.

4 Un número del PMI por encima de 50 indica una expansión en la actividad del sector, mientras que por debajo indica una contracción.
} 


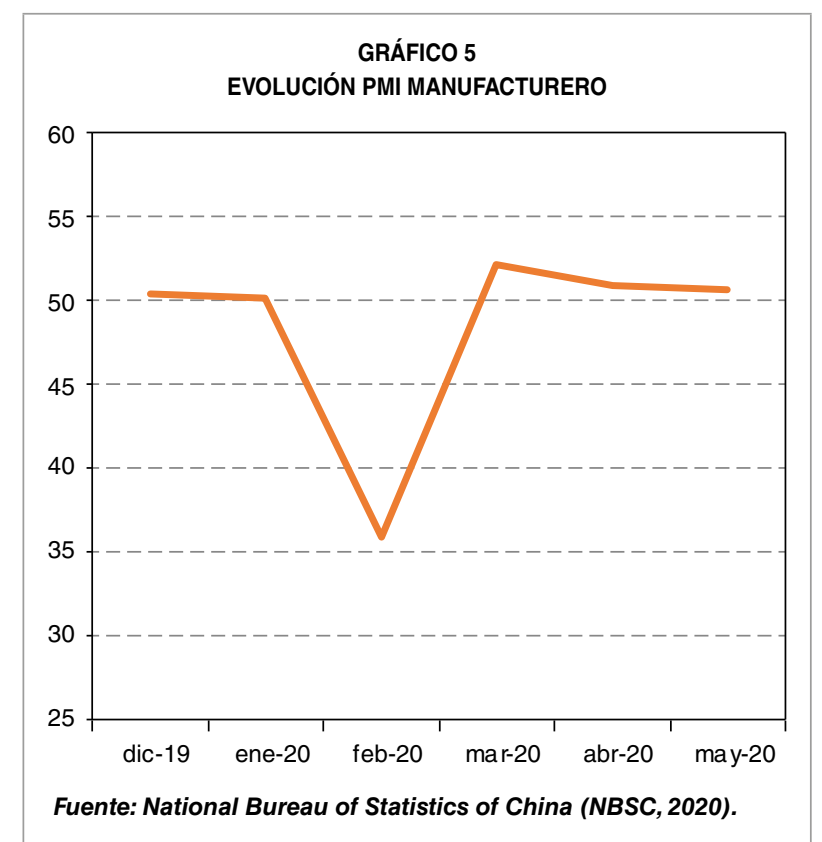

se debió, principalmente, a la relajación gradual de las restricciones, a la movilidad de personas y mercancías dentro de China que obligaron a una reincorporación por etapas de los trabajadores a los centros de producción (Chu et al., 2020) y a los problemas logísticos en el abastecimiento de materias primas y suministro de bienes al exterior por el cierre de fronteras de los países tras la propagación de la COVID-19 a nivel mundial (Chen et al., 2020). Prueba de ello es que a finales de abril el $98 \%$ de las grandes empresas había reanudado su actividad, pero solo el $77,3 \%$ operaba por encima del $80 \%$ de su capacidad productiva (Tan, 2020).

El sector servicios, por su parte, se contrajo un $5,2 \%$ en el primer trimestre como consecuencia de la paralización del transporte y el cierre de las atracciones turísticas, establecimientos, centros de ocio y comercios. El índice de gestores de compras (PMI) no manufacturero, indicador del sentimiento de mercado en los sectores de servicios y la construcción, se contrajo hasta los 29,6 puntos en febrero,

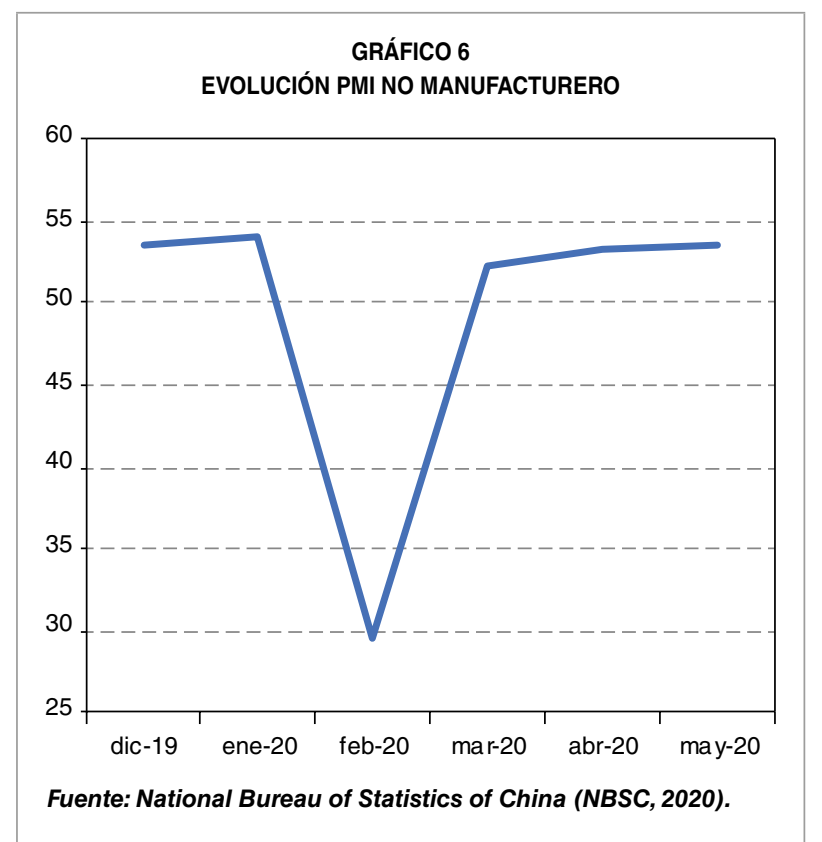

recuperándose en marzo y abril con 52,3 y 53,2 puntos, respectivamente (Gráfico 6). La recuperación del PMI no manufacturero en abril vino liderada por su índice de la construcción, que se situó en 59,7 puntos, un aumento de 4,6 puntos con respecto a marzo (NBSC, 2020), gracias a la reactivación de los proyectos públicos de infraestructuras (Cui, 2020). En mayo el PMI no manufacturero ascendió a 53,6 puntos (NBSC, 2020).

\subsection{Impacto en la coyuntura económica}

En definitiva, desde principios de marzo la economía china ha experimentado un progresivo proceso de reactivación que se ha prolongado hasta el mes de mayo. Durante este tiempo, como se ha explicado en los apartados anteriores, la reacción de los agentes económicos ha sido dispar, mientras que las empresas reaccionaban con rapidez y en el mes de abril se situaban de nuevo en terreno positivo, los consumidores han mantenido una actitud $\triangleright$ 
más cautelosa (Gráficos 2 y 3). Esta reactivación de la actividad industrial a mayor velocidad que la recuperación de la demanda interna, junto con el abrupto descenso de la demanda externa a partir del mes de marzo por el cierre total o parcial del resto de países para hacer frente a la epidemia de la COVID-19, han dado lugar a la aparición de presiones deflacionistas en la economía china.

En concreto, la caída del consumo interno ha provocado una desaceleración de los precios al consumo (IPC), que han pasado del 5,2\% interanual en febrero, al 3,3\% en abril y al $2,4 \%$ en mayo. Por su parte, el exceso de oferta ha presionado a la baja el índice de precios al productor (IPP), experimentando una caída del 0,5\% interanual en febrero, que se ha agudizado con una reducción del 3,1\% interanual en abril y del $3,5 \%$ en mayo (NBSC, 2020).

Estas tensiones deflacionistas han provocado una reducción de los ingresos de las empresas y, en consecuencia, de los beneficios empresariales del $-38,7 \%$ interanual en el periodo enero-febrero, seguido del $-36,7 \%$ en el primer trimestre y del $-27,4 \%$ en los cuatro primeros meses del año (NBSC, 2020).

No obstante, el impacto de la crisis de la COVID-19 está siendo distinto según el tipo de empresa. Las empresas más afectadas por la paralización de la actividad económica y la contracción de la demanda han sido las pequeñas y medianas empresas (pymes), creadoras del $80 \%$ del empleo y el $60 \%$ del PIB en China (Holzmann y Kärnfelt, 2020), al experimentar problemas derivados de la falta de liquidez por la reducción de las ventas y acumulación de stocks, así como la falta de trabajadores que no podían reincorporarse a sus lugares de trabajo, lo que ha obligado a muchas a reducir su actividad o a permanecer cerradas (Zhuang, 2020). Con base en lo anterior, un estudio de la
Universidad de Tsinghua (Pekín) estimó que más del $70 \%$ de las pymes encuestadas esperaban impactos negativos significativos en sus ingresos a finales de marzo y que el $85 \%$ no podría sobrevivir más de tres meses sin respaldo financiero (Zhu et al., 2020).

Por el contrario, la situación de las grandes empresas de propiedad estatal ha resultado menos grave al contar con un mayor acceso a financiación, disfrutar de un amplio colchón de reservas en efectivo y la protección del Gobierno, lo que les permitió acelerar su vuelta a la actividad a lo largo del mes de marzo (Holzmann y Kärnfelt, 2020). Fruto de ello, mientras el valor agregado industrial de las empresas privadas se contrajo el $11,3 \%$ en el primer trimestre, en las empresas estatales esta reducción se situó en el - $6 \%$ interanual (NBSC, 2020).

Con independencia del impacto de la crisis por tipo de empresa, el descenso de los ingresos empresariales ha sido generalizado, obligando a muchas compañías a una reducción de gastos para equilibrar sus cuentas que, en gran medida, se está realizando mediante ajustes en la masa salarial o el despido de trabajadores. En los primeros meses de 2020, las pérdidas salariales de los trabajadores migrantes se estimaron en 115.000 millones de dólares (Leng, 2020).

Pese a estos esfuerzos, muchas empresas no han sido capaces de equilibrar su situación financiera, viéndose obligadas a reducir o cesar su actividad. A finales de marzo, casi 500.000 empresas chinas fueron dadas de baja, mientras que la creación de nuevas empresas se redujo en más de un 30\% en comparación con 2019 (Holzmann y Kärnfelt, 2020).

Consecuentemente, la tasa de desempleo urbano, variable de referencia que usan las autoridades chinas, se ha incrementado $D$ 
desde el $5,2 \%$ de enero al $6 \%$ en abril (NBSC, 2020). De acuerdo con algunas estimaciones no oficiales, en los meses de marzo y abril la tasa de desempleo se habría situado entre el $11 \%$ y el $20 \%$ en los sectores secundario y terciario, lo que equivaldría a una destrucción de entre 60 y 100 millones de puestos de trabajo. Teniendo en cuenta el compromiso oficial de las compañías estatales de no reducir sus plantillas durante la crisis, la mayoría de estos despidos procederían de empresas privadas de los sectores de la construcción (23 millones de desempleados), el comercio minorista (30 millones de desempleados) y la hostelería y el catering (14 millones de desempleados) (Cui, 2020).

Por este motivo, como se describe en el siguiente apartado, la estabilización del mercado de trabajo se ha convertido en el principal motivo de preocupación de las autoridades en su gestión de la recuperación económica del país tras la crisis de la COVID-19.

\section{Políticas económicas frente a la COVID-19}

Ante el desplome histórico de la economía china por las medidas de control en la lucha contra la COVID-19, las autoridades no han anunciado ningún plan integral de estímulo económico, como ocurrió durante la crisis financiera de 2008. Desde el inicio de la epidemia, China ha optado por un enfoque conservador basado en la aplicación moderada de políticas económicas expansivas para dar apoyo inmediato a los agentes afectados y amortiguar el impacto en el crecimiento del país.

La celebración del 21 al 28 de mayo de las «Dos Sesiones» sirvió al Gobierno chino para respaldar oficialmente la continuidad de las políticas fiscales y monetarias aplicadas hasta la fecha, con la prioridad puesta en la estabilización del mercado laboral, en detrimento de un objetivo de crecimiento, como venía siendo tradicional desde 1990.

Desde el inicio de la epidemia la estrategia de política monetaria del Banco Central de China $(\mathrm{PBoC})$ se ha centrado principalmente en tres objetivos. En primer lugar, el incremento del nivel de liquidez en el sistema con tres recortes en el coeficiente legal de caja de los bancos. En segundo lugar, la suavización de las condiciones para la concesión de crédito y el abaratamiento de los préstamos a las empresas más afectadas por la epidemia. En este sentido, la tasa de los préstamos a medio plazo (un año) para instituciones financieras ha pasado del 3,25\% a principios de año al 2,95\% en mayo, mientras que la tasa preferencial de préstamos a un año ha caído 30 puntos básicos, hasta alcanzar el 3,85\% (PBoC, 2020a y 2020b). Por último, el alivio de los problemas de liquidez de las pymes mediante recomendaciones a las instituciones financieras sobre la adopción de soluciones de crédito innovadoras, la optimización de los procedimientos de aprobación de préstamos (Administración Estatal de Impuestos, 2020) y un aumento del $40 \%$ en los préstamos concedidos por los bancos comerciales a pymes y microempresas (Keqiang, 2020).

En materia fiscal, el Gobierno ha aprobado medidas para la estabilización del empleo y la reducción temporal de cargas a las empresas, con especial atención a las pymes. Destaca el anuncio del Gobierno, a finales de mayo, de prolongar hasta final de año los recortes de impuestos y tasas para empresas, por valor aproximado de 70.000 millones de dólares, promulgadas en la fase anterior y cuya vigencia terminaba en junio. Estas medidas incluyen $\triangleright$ 
la exención a pymes y microempresas del pago de las primas de los seguros de vejez, desempleo y accidentes laborales de la Seguridad Social, la exención parcial o total del IVA a los pequeños contribuyentes; la exención del IVA a servicios como transporte público, restauración y hostelería, turismo y ocio, cultura y deporte. El pago del impuesto de sociedades por parte de las pymes y los autónomos queda aplazado sin excepción hasta el 2021. Asimismo, se ha tratado de fortalecer el apoyo al sector sanitario con la asignación, a principios de marzo, de un fondo de 15.920 millones de dólares que incluye subsidios al personal médico y estímulos fiscales a los fabricantes de materiales de prevención y control de epidemias (Ministerio de Hacienda, 2020a). Finalmente, el anuncio de la ampliación del déficit público desde el 2,8\% hasta el 3,6\% del PIB para 2020 durante las «Dos Sesiones» indica la clara intención de las autoridades chinas de continuar aprobando nuevos estímulos fiscales a nivel nacional y local en función de la evolución del control de la epidemia y la crisis económica mundial (Keqiang, 2020).

Respecto a las autoridades provinciales, desde principios de año se ha ido incrementando de manera sustancial la cuota de emisión de los denominados «bonos de propósito especial» hasta alcanzar a finales de mayo los 525.000 millones de dólares, un incremento de 224.000 millones de dólares con respecto a 2019. Estos bonos están destinados a la financiación de proyectos en nueva infraestructura (p.e., internet de las cosas, 5G, inteligencia artificial), urbanismo, energía y salud pública. Las provincias menos endeudadas han lanzado campañas locales de emisión de cupones de consumo digital por valor de 2.700 millones de dólares a través de las plataformas de pago móviles más importantes del país para estimular el consumo en turismo, comercio minorista, entretenimiento y restauración (Shen et al., 2020). Destaca, por último, la aprobación

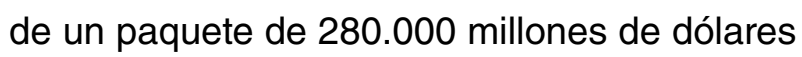
para estimular el gasto público local (Keqiang, 2020).

A nivel sectorial, desde finales de febrero, el Gobierno está implementando iniciativas para reactivar la actividad en determinadas industrias. De las distintas medidas aprobadas cabe destacar el apoyo financiero que ofrece desde principios de marzo la Administración de Aviación Civil de China (CAAC) a las aerolíneas nacionales y extranjeras que no suspendan o que reanuden sus vuelos internacionales con origen y/o destino China (Ministerio de Hacienda, 2020b). Asimismo, a mediados de abril se incrementaron los subsidios para préstamos garantizados a determinadas pymes que emprendan nuevos negocios o trabajen en el sector mayorista, minorista y de restauración, así como a los conductores a tiempo completo que han pedido préstamos para la compra de automóviles para cubrir el servicio en línea (Ministerio de Hacienda, 2020c). Por último, a finales de abril se aprobó la ampliación de la exención a los vehículos de nueva energía (NEV) del impuesto de compra de vehículos y de los subsidios a determinados NEV hasta el 2022 (Ministerio de Hacienda y Ministerio de Industria y Tecnología de la Información, 2020).

En definitiva, las autoridades chinas son conscientes de la necesidad de actuar de manera decidida para contrarrestar los problemas derivados de la COVID-19, aunque a través de un enfoque diferente a otras crisis, al centrarse en medidas específicas dirigidas a estimular a aquellos sectores productivos que ofrezcan un mayor valor añadido, estabilizar el mercado laboral y proteger a aquellos sectores de la sociedad más desfavorecidos. De esta manera, $D$ 
China está desplegando un programa de políticas económicas mucho más eficiente que se irá ajustando en función de la evolución de la crisis económica y el control de la epidemia de la COVID-19.

\section{Conclusiones}

La crisis de la COVID-19 ha generado un doble shock negativo de oferta y demanda en la economía china durante el primer trimestre de 2020, que ha provocado una caída del consumo y la inversión doméstica, y una parálisis de la actividad industrial sin precedentes. Desde mediados del mes de febrero, la actividad empresarial se está recuperando a buen ritmo, mientras que la demanda interna está experimentando una recuperación más paulatina; a esto hay que añadir que la demanda externa se ha congelado tras la expansión de la COVID-19 a nivel mundial. Esta distinta evolución de la demanda y la oferta está generando un entorno deflacionista en la economía china que ha impactado negativamente en los beneficios empresariales, obligando a las empresas a reducir sus plantillas y, eventualmente, abocándolas al cierre.

Por este motivo, las autoridades chinas, que han monitorizado muy de cerca el impacto de la COVID-19 y adoptado constantes medidas de estímulo, han fijado como principal objetivo de política económica en 2020 la estabilidad del mercado laboral en detrimento de lograr un objetivo concreto de crecimiento económico, como había sido habitual desde 1990.

Pese a los importantes desafíos pendientes, la evolución de las magnitudes económicas chinas en los meses de abril y mayo muestran que este país se encuentra en pleno proceso de recuperación, confirmando las previsiones de abril del FMI que apuntaban a que sería el único Estado en experimentar una tasa de crecimiento positiva en 2020 , junto a India. No obstante, esta recuperación dependerá en buena medida de la velocidad a la que se logre controlar la epidemia a nivel mundial y del éxito en evitar la aparición de nuevos rebrotes de la enfermedad.

Con independencia de la evolución de estos factores, resulta evidente que China ha logrado en un plazo de poco más de cinco meses controlar la evolución de la epidemia e iniciar el proceso de reactivación de su economía hasta permitirle regresar a una cierta normalidad en la vida diaria de la población. Por este motivo, esta evolución puede tomarse como una referencia y, especialmente, como una señal de optimismo para la superación de la crisis.

\section{Bibliografía}

Administración Estatal de Impuestos (2020). Aviso sobre el papel de la interacción del impuesto al dinero para ayudar a las pequeñas y microempresas a reanudar el trabajo. Circular número 10. Comisión Reguladora de Banca y Seguros de China y Administración Estatal de Impuestos. Pekín. China.

Baijie, A. (11 de marzo de 2020). Xi vows victory over coronavirus in Wuhan. Chinadaily. Pekín. China.

CAAM (2020a). Economic performance of Automobile Industry in March 2020. China Association of Automobile Manufacturers. Pekín. China.

CAAM (2020b). Economic Performance of Automobile Industry in April 2020. China Association of Automobile Manufacturers. Pekín. China.

Chen, S., Zhao, Y., y Han, M. (30 de abril de 2020). China Factory Data Show Global Slump Weaken Nascent Recovery. Bloomberg News. Nueva York. USA. 
Cheng, S. (17 de mayo de 2020). Masks outdoors no longer required, authorities say. Chinadaily. Pekín. China.

Cheng, Y. (2020). Over $60 \%$ of SMEs outside Hubei resume work, Chinadaily. Pekín. China. Publicado: 13 de marzo de 2020.

Chu Y., Gao, W., Chen, A., Yin, X., y Wang, C. (21 de marzo de 2020). Roaring Chinese factories in full swing to stabilize global supply chain. Xinhua. Pekín. China.

Consejo de Estado de China (9 de febrero de 2020a). Mecanismo conjunto de prevención y control del Consejo de Estado. Pekín. China.

Consejo de Estado de China (7 de mayo de 2020). Mecanismo conjunto de prevención y control sobre cómo salir bien de la nueva epidemia de neumonía coronaria. Circular número 14. Pekín. China.

Cui, E. (25 de mayo de 2020). The Truth About Unemployment. Gavekal Dragonomics. Hong Kong. China.

García Herrero, A., Xu, J., y Ng, G. (9 de abril de 2020). COVID-19 Uncovers China's Household Debt Accumulation and Its Risks. Natixis. Hong Kong. China.

Gobierno Provincial de Hubei (2020, 23 de enero). Circular de la nueva sede de prevención y control de la neumonía por infección por coronavirus en Wuhan. Circular número 1. Wuhan. China.

Holzmann, A., y Kärnfelt, M. (12 de mayo de 2020). Supporting China's SMEs affected by COVID-19 is crucial to avoid a socioeconomic disaster. The Mercator Institute for China Studies (MERICS). Berlín. Alemania.

IMF (2020). The Great Lockdown. En World Economic Outlook. Abril (Capítulo 1, página 8). International Monetary Fund. Washington DC. EE UU.

Keqiang, L. (22 de mayo de 2020). Informe sobre la Labor del Gobierno. III Sesión de la XIII Asamblea Popular Nacional. Consejo de Estado. Pekín. China.
La Monica, P.R. (22 de mayo de 2020). The way we shop has fundamentally changed. That's good news for Alibaba. CNN. Atlanta, Georgia. EE UU.

Lee, A. (25 de enero de 2020). The economic importance of China's Lunar New Year as the Year of the Rat begins. South China Morning Post. Hong Kong. China.

Leng, S. (6 de marzo de 2020). China's huge migrant worker population bearing the brunt of economic shutdown. South China Morning Post. Hong Kong. China.

Mazor-Freedman, A. (27 de abril de 2020). In China, Offline Retail Isn't Going Back to Normal. Gartner. Stamford, CT. EE UU.

Ministerio de Hacienda (5 de marzo de 2020a). Conferencia de trabajo para responder eficazmente al impacto de la epidemia y fortalecer los trabajos financieros locales de «tres garantías». Pekín. China.

Ministerio de Hacienda (4 de marzo de 2020b). Aviso sobre la política de apoyo financiero durante la prevención y el control de la nueva epidemia de coronavirus en empresas de transporte de aviación civil. Circular número 30. Pekín. China.

Ministerio de Hacienda (15 de abril de 2020c). Aviso sobre el aumento adicional del descuento de intereses de los préstamos garantizados de emprendimiento y el pleno apoyo al emprendimiento y el empleo de grupos clave. Circular número 21. Pekín. China.

Ministerio de Hacienda y Ministerio de Industria y Tecnología de la Información (16 de abril de 2020). Anuncio sobre las políticas relativas a la exención del impuesto sobre la compra de vehículos en vehículos de nueva energía. Circular número 21. Pekín. China.

Municipalidad de Pekín (31 de enero de 2020a). Aviso del Gobierno Popular Municipal de Pekín sobre disposiciones flexibles para las empresas de este municipio durante la prevención y el control de la neumonía infectada por el nuevo coronavirus. Circular número 3. Pekín. China. $\triangleright$ 
Municipalidad de Pekín (15 de febrero de 2020c). Todo el personal que regresa a Pekín debe quedarse en casa o concentrarse en la observación durante catorce días. Pekín. China.

NBSC (National Bureau of Statistics of China) (2020). Press Release (varias). Pekín. China. http://www.stats.go v.cn/english/

Organización Mundial de la Salud (27 de abril de 2020a). COVID-19: cronología de la actuación de la OMS. Centro de Prensa. Ginebra, Suiza.

Organización Mundial de la Salud (2020b). WHO Coronavirus Disease (COVID-19) Dashboard. Ginebra, Suiza. https://COVID-19.who.int/

PBoC (Banco Central de la R.P. de China) (15 de abril de 2020). Open Market Operations, n.․71. Pekín. China.

PBoC (Banco Central de la R.P. de China) (20 de abril de 2020b). Announcement on Loan Prime Rate. Pekín. China.
Ren, Y. (14 de marzo de 2020). Europe becomes epicenter of pandemic. Global Times. Pekín. China.

Shen, F., Cheng, S., Yu, H., y Guo, Y. (19 de marzo de 2020). Vouchers to Boost Post-Virus Spending Trigger Affordability Warning, Caixin. Pekín. China.

Tan, H. (29 de abril de 2020). Slowing global demand due to coronavirus hits China's manufacturing sector in April, two sets of data show. CNBC. Nueva Jersey. EE UU.

Wang, C. (5 de mayo de 2020). Holiday economy rebounds. Global Times. Pekín. China.

Zhu, W., Liu J., Wei W., y Ouyang L. (2020). COVID-19 and Impacts on SMEs: Survey Evidences. CEIBS Business Review. Shanghai. China.

Zhuang, A. Y. (2 de abril de 2020). China View: The long road to recovery. Nordea Markets. Helsinki. Finlandia. 
